\title{
CAPACITANCE SPECTROSCOPY OF SINGLE-BARRIER GaAs/AlAs/GaAs STRUCTURES CONTAINING InAs QUANTUM DOTS
}

\author{
A.E. BeLYAEV \\ Institute of Semiconductor Physics, NASU, Kiev 252028, Ukraine \\ L. Eaves, P.C. Main, A. Polimeni, S.T. Stoddart and M. Henini \\ Department of Physics, University of Nottingham, Nottingham, NG7 2RD, U.K.
}

An electrostatic profile of single-barrier heterosizuctures with InAs quantum dots encased into barrier has been studied. The role of growth conditions and structure's design is investigated. The charging state and position of energy levels for InAs quantum dots embedded in AlAs matrix are discussed.

PACS numbers: $73.40 . \mathrm{Gk}, 85.30 . \mathrm{Mn}$

In this paper we investigate the capacitance-voltage, $C(V)$, and magneto-capacitance, $C(B)$, characteristics of a series of single-barrier $n-i-n$ GaAs/AlAs/GaAs heterostructures incorporating a layer of self-assembled InAs quantum dots (QDs) in the AlAs barrier. Thicknesses of the layers of AlAs deposited before and after the InAs QD growth are varied. The $C(V)$ and $C(B)$ results are compared with those from a control sample that has no InAs and a sample in which the InAs thickness is such that a continuous wetting layer (WL) of InAs is grown.

The samples presented in this study were grown on a (100) $n^{+}$-GaAs substrate using MBE and all consist of $n$-type GaAs devices with an AlAs tunnel barrier region surrounded by $100 \mathrm{~nm}$ not intentionally doped spacers to minimise the effect of dopant diffusion into the central barrier region. These spacers are then enclosed by $100 \mathrm{~nm}$ of $n$-type GaAs doped to a density of $10^{16} \mathrm{~cm}^{-3}$ lightly doped region, followed by $100 \mathrm{~nm}$ doped to $10^{17} \mathrm{~cm}^{-3}$, and then a heavily doped contact layer, at $10^{18} \mathrm{~cm}^{-3}$. The control sample consists of a $10 \mathrm{~nm}$ AlAs single barrier, while the WL sample consists of an AlAs double barrier structure enclosing 1.4 ML of InAs. The QD samples consist of a InAs QD layer enclosed in AlAs layers grown previously and subsequently to the QD growth. The QDs are produced by deposition of $1.8 \mathrm{ML}$ of InAs at a growth temperature of $520^{\circ} \mathrm{C}$. The detailed layer make-up of the samples presented in this work are shown in Table.

The band diagram of the samples at zero voltage and under applied bias is drawn schematically in Fig. 1. Electrical contacts were formed on the doped GaAs 
TABLE

The layer composition of InAs QD samples and the control sample.

\begin{tabular}{c|c|c|c}
\hline \hline Sample & Underlayer [nm] & InAs layer [ML] & Overlayer [nm] \\
\hline $\mathrm{A}$ & 5.1 & 1.8 & 5.1 \\
$\mathrm{~B}$ & 3.4 & 1.8 & 6.4 \\
$\ddot{\mathrm{C}}$ & 5.1 & 1.8 & 7.2 \\
$\mathrm{D}$ & 5.1 & 1.8 & 3.8 \\
WL & 5.1 & 1.4 & 5.1 \\
Control & 10 nm AlAs & no QDs & \\
& single barrier & &
\end{tabular}

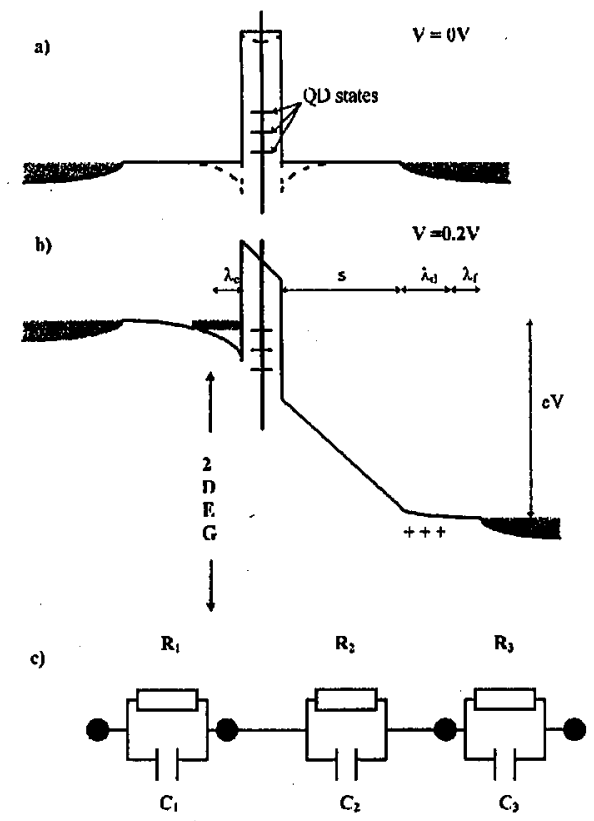

Fig. 1. Schematic band diagrams of the device at zero bias (a) and $0.2 \mathrm{~V}$ (b), and equivalent electric circuit of the device (c).

layer by AuGe alloying, and devices were fabricated into mesas of $200 \mu \mathrm{m}$ diameter. In this paper the convention of negative substrate for forward bias is used. The measurements of capacitance were all made using a HP 4275A Multi-Frequency LCR Meter, in a variable temperature cryostat. The $C(V)$ analyser is capable of measuring in the frequency range of $10 \mathrm{kHz}-10 \mathrm{MHz}$.

Figure 2 shows the capacitance-voltage curves of the samples nominated in Table. The values of the WL and QD sample curves have each been displaced by $4 \mathrm{pF}$ for clarity. This measurement treats the sample as if it were a parallel 


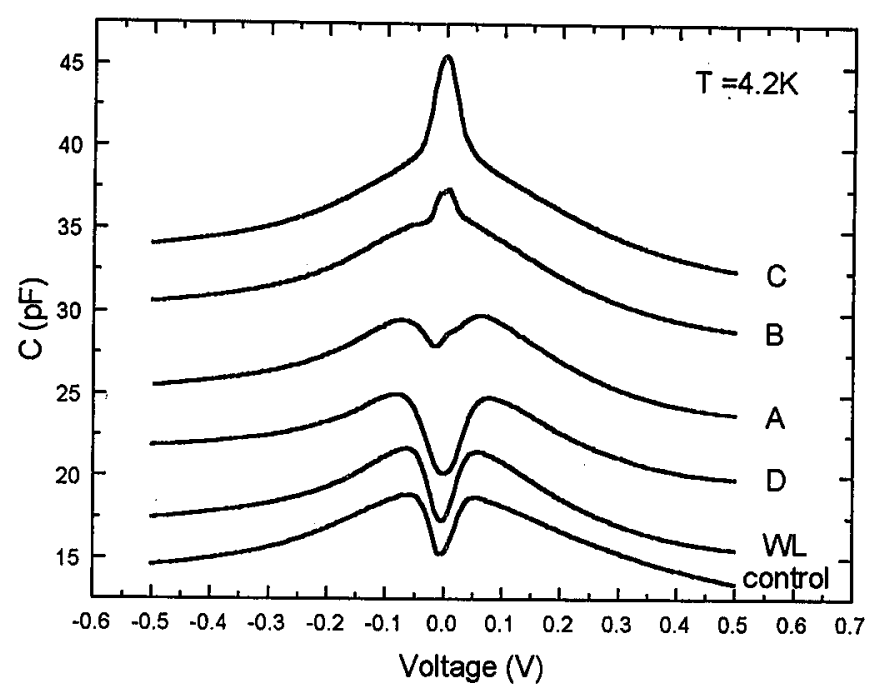

Fig. 2. Capacitance-voltage curves for the various devices taken at $4.2 \mathrm{~K}$ and zero magnetic field. Top five curves are displaced vertically by values of $\Delta C=4 \mathrm{pF}$ each for clarity.

combination of impedance, $R$, and single capacitance, $C$. It can be seen that there is no clear partition between QD and non-QD samples apparent from the $C(V)$. All the $C(V)$ characteristics share a common form for biases above ca. $200 \mathrm{mV}$, characterised by a declining capacitance. This is to be expected as the capacitance is simply dominated by that of the depleted contact region for all the samples. The most variance seen in the samples is in the form of $C(V)$ around zero voltage. There are four samples that exhibit a pronounced dip in $C(V)$, while two of QD samples show a pronounced peak at zero bias. To understand the origin of the form of the $C(V)$ we will follow the arguments described below. If there is no net charge in the barrier (or there is a small negative charge) at zero bias, then at low bias voltages we have accumulation on the left hand side and depletion on the right hand side. In this case resistances $R_{1}, R_{3}$ more or less short circuit $C_{1}, C_{3}$. Also $R_{2} \approx \infty$ since AlAs barrier is thick and high. So we are mainly concerned with behaviour of $C_{2}$. The areal capacitance $C_{2}$ equals to $\varepsilon_{r} \varepsilon_{0} / d$. Here $d=\lambda_{\mathrm{e}}+b+s+\lambda_{\mathrm{d}}$, where $\lambda_{\mathrm{e}}$ is the quantum stand-off distance of the electrons in the 2DEG, $s$ is the undoped spacer layer thickness, $\lambda_{d}$ is the depletion layer width and $\lambda_{f}$ is the width of the lightly doped contact layer, see Fig. 1b. Thus, $C_{2}$ will strongly depend on variation of $\lambda_{e}$ and $\lambda_{\mathrm{d}}$. The Fang-Howard theory [1] gives $\lambda_{\mathrm{e}} \sim n_{s}^{-1 / 3}$ and $\lambda_{\mathrm{d}} \sim n_{s}$, where $n_{s} e$ is areal charge in the left hand side of $2 \mathrm{DEG}$. So, at low $n_{s} 1 / C_{2}$ is proportional to $n_{s}^{-1 / 3}$, but at high $n_{s} 1 / C_{2}$ gradually increases with $n_{s}$. This shows qualitatively that there is a maximum in $C_{2}$, and consequently in measured capacitance, at finite $n_{s}$ (non-zero voltage) and minimum at zero voltage. The control sample has a clear minimum in capacitance at $0 \mathrm{~V}$, a feature that is shared by the wetting layer sample, and QD samples A and D. Therefore the similarity in the capacitance 
values of the low bias $C(V)$ leads to the conclusion that samples $\mathrm{A}$ and $\mathrm{D}$, and the control sample as well as the WL sample are very similar electrostatically, and that the QD-containing barrier in samples $A$ and $D$ is almost electrically neutral. If we assume that samples have a small positive charge in the barrier (associated with QDs), then at zero bias both contacts are accumulated. Under these conditions middle capacitance $C_{2}$ is given by the expression: $1 / C_{2} \sim \lambda_{\mathrm{e} 1}+b+\lambda_{\mathrm{e} 2}$, where $\lambda_{\mathrm{e} 1}$ and $\lambda_{\mathrm{e} 2}$ are the quantum stand-off distances of the electrons in the 2DEG formed in front and at rear of the barrier. Both $\lambda_{\mathrm{e} 1}$ and $\lambda_{\mathrm{e} 2}$ will be determined by sheet density of the 2DEG, i.e., $\lambda_{\mathrm{e} 1} \sim n_{\mathrm{s} 1}^{-1 / 3}$ and $\lambda_{\mathrm{e} 2} \sim n_{\mathrm{s} 2}^{-1 / 3}$, and $n_{s 1}+n_{s 2}=N_{0}$, where $N_{0}$ is the net positive charge density concentrated in the barrier. A simple algebra shows that in the case of $n_{s 1}=n_{s 2}$ capacitance $C_{2}$ has a maximum at zero bias. The behaviour of $C(V)$ characteristics around zero voltage for samples $\mathrm{B}$ and $\mathrm{C}$ is consistent with this interpretation, thus confirming the presence of a net positive charge in the $\mathrm{QD}$-containing barrier.

In order to probe the structures further, magneto-capacitance measurements were performed at $4 \mathrm{~K}$ upon the samples. These entailed sweeping a magnetic field $B$, applied parallel to the growth direction, up to $\approx 10 \mathrm{~T}$ for a given bias voltage. This results in familiar Shubnikov-de Haas-like magneto-oscillations in the capacitance with a periodicity in $1 / B$, due to the Landau levels in the accumulation layer passing through the Fermi energy [2]. The charge modulation arising in this case affects the distribution of electric potential and screening length, and hence modulates the capacitance of the device. The frequency of the oscillations of fundamental field, $B_{\mathrm{f}}=[\Delta(1 / B)]^{-1}$, is thus related to the Fermi energy $E_{\mathrm{F}}$ by $B_{\mathrm{f}}=2 \pi m^{*} E_{\mathrm{F}} / e h$, where $m^{*}$ and $h$ are the effective mass and the Plank constant, respectively. The $B_{\mathrm{f}}$ therefore measures the electronic sheet density of 2DEG in the accumulation layer $n_{s}=2 e B_{\mathrm{f}} / h$. Sheet density at zero voltage determined by such a manner for all samples is shown in Fig. 3, where the extracted $\Delta n_{s}$ values are plotted versus $C(0)$. The result confirms our explanation of the zero-field $C(V)$ at low temperatures.

In conclusion, we investigate the $C(V)$ and $C(B)$ characteristics of a series of single-barrier $n-i-n$ GaAs/AlAs/GaAs heterostructures incorporating a layer of self-assembled InAs quantum dots in the AlAs barrier. Some of the samples show minima and some maxima in the zero-field $C(V)$ at low temperatures. This provides information about the degree of accumulation in the GaAs layers adjacent to the barrier at zero bias. We have shown that the control sample exhibits the same behaviour as some QD samples, implying electrical neutrality in the QD-containing barrier of these samples. Other samples are seen to have a slight surfeit of positive charge, leading to electron accumulation at zero bias. There are two interesting consequences of this arrangement. The first is that there are electrons brought into intimate contact with the QDs at zero applied bias. The coupling of the electrons to the QDs may be enhanced by this at low biases, and may even lead to interesting zero-bias tunneling effects. Another consequence is that the positive charge in the QD-containing barrier radically alters the picture of the band bending in the devices coming from a model consideration of only the charging QD states with electrons. 


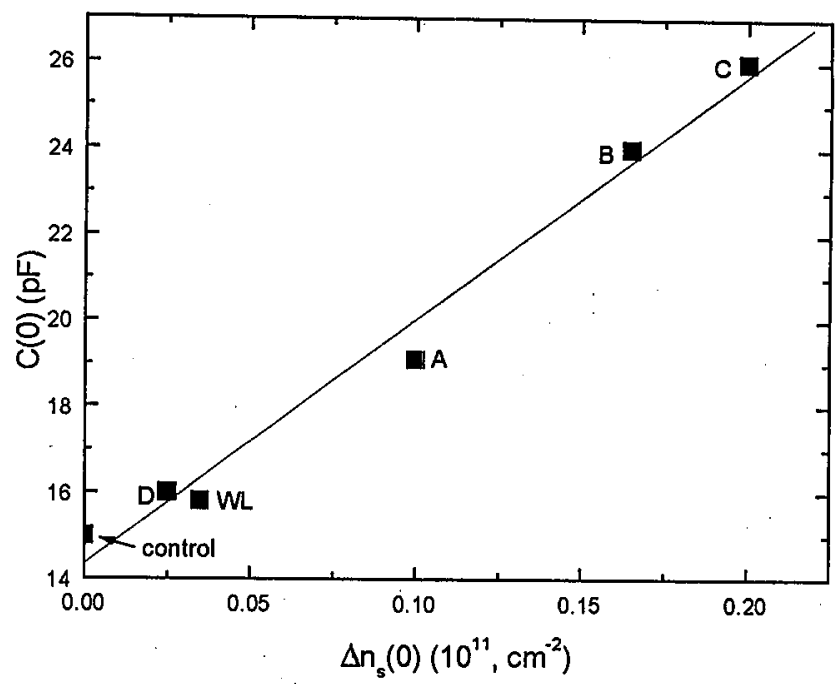

Fig. 3. The values of capacitance at zero voltage versus $\Delta n_{s}$. Solid line presents least-squares fit.

This work is supported by EPSRC (U.K.). A.E. Belyaev and L. Eaves acknowledge the Royal Society and EPSRC for financial support respectively.

\section{References}

[1] F.F. Fang, W.E. Howard, Phys. Rev. Lett. 16, 797 (1966).

[2] M.L.L. Leadbeater, E.AS. Alves, F.W. Sheard, L. Eaves, M. Henini, O.H. Hughes, G.A. Toombs, J. Phys., Condens. Matter 1, 10605 (1989). 